\title{
Subwavelength Topological Edge States in Optically Resonant Dielectric Structures
}

\author{
Alexey P. Slobozhanyuk, ${ }^{1,2}$ Alexander N. Poddubny, ${ }^{2,3}$ Andrey E. Miroshnichenko, ${ }^{1}$ Pavel A. Belov, ${ }^{2}$ and Yuri S. Kivshar ${ }^{1,2}$ \\ ${ }^{1}$ Nonlinear Physics Center, Australian National University, Canberra ACT 0200, Australia \\ ${ }^{2}$ ITMO University, Saint Petersburg 197101, Russia \\ ${ }^{3}$ Ioffe Physical-Technical Institute of the Russian Academy of Science, Saint Petersburg 194021, Russia
}

(Received 29 October 2014; published 24 March 2015)

\begin{abstract}
We suggest a novel type of photonic topological edge states in zigzag arrays of dielectric nanoparticles based on optically induced magnetic Mie resonances. We verify our general concept by the proof-ofprinciple microwave experiments with dielectric spherical particles, and demonstrate, experimentally, the ability to control the subwavelength topologically protected electromagnetic edge modes by changing the polarization of the incident wave.
\end{abstract}

DOI: 10.1103/PhysRevLett.114.123901

PACS numbers: 42.70.Qs, 42.79.Fm, 42.79.Gn, 42.79.Ta

Topological insulators represent a novel class of materials with a topologically protected phase order [1]. Such materials attracted a lot of attention in the past due to the existence of novel types of conducting surface states in, otherwise, insulating bulk materials being protected by time-reversal symmetry, and they demonstrate exotic phenomena such as the quantum Hall effect $[2,3]$. Recently, the concept of topological insulators became attractive in optics, and different types of electromagnetic topological states have been realized for microwave and photonic systems [4]. In particular, the recent suggestions include breaking the time-reversal symmetry by applying a periodic modulation to the structure [5] and engineering photonic crystals and metamaterials with synthetic magnetic fields and spin-orbit interactions [6,7]. The first electromagnetic topological edge states were demonstrated for radio frequencies [8-10], and such novel photonic states were also realized at optical frequencies in coupled optical waveguides [11,12] and optical lattices [13,14].

Recently, it was predicted theoretically that a novel type of topological edge states can be realized in the subwavelength regime for a zigzag chain of plasmonic nanoparticles [15]. Nontrivial topological properties of these plasmonic edge states have been studied in the framework of the coupled-dipole approximation and, also, by direct numerical simulations of Maxwell's equations.

The purpose of this Letter is twofold. First, we demonstrate, both theoretically and experimentally, that the concept of plasmonic topological edge states in zigzag arrays introduced earlier for metallic nanoparticles [15] is, in fact, much more general, and it can be employed for a broader class of subwavelength photonic and electromagnetic structures including arrays of dielectric nanoparticles with electric and magnetic Mie resonances, recently suggested as building blocks of all-dielectric metamaterials. More importantly, due to this generality, the subwavelength structures with a topological order can be observed for different types of coupled optical modes, including optically induced magnetic quadrupole resonant modes. Second, we employ microwave experiments with zigzag arrays of electromagnetically coupled dielectric spheres and provide the first proof-of-principle experimental observation of optically induced magnetic topological edge states operating in the subwavelength regime. We demonstrate that such topological edge states can be selectively excited with the linear polarization of the incident electromagnetic wave, and we visualize them directly by mapping the corresponding electromagnetic fields. Furthermore, we demonstrate the difference between topologically trivial and nontrivial electromagnetic modes in this model. Importantly, in a sharp contrast to plasmonic arrays [15], the optically induced response of dielectric nanoparticles includes both electric and magnetic resonances which do not suffer from strong losses in metals at visible frequencies, so that the dielectric structures can be employed for the realization of a large variety of novel low-loss nanophotonic devices with superior characteristics [16] and topological properties.

First, we start from the discussion of topological properties of a general model originating from two major assumptions: (i) polarization-dependent interaction between resonant modes of its structural elements, and (ii) a zigzag shape of the array [Fig. 1(a)]. The structure Hamiltonian reads

$$
H=\sum_{j, \nu} E_{0} a_{j \nu}^{\dagger} a_{j \nu}+\sum_{\left\langle j, j^{\prime}\right\rangle, \nu, \nu^{\prime}} a_{j \nu}^{\dagger} V_{\nu \nu^{\prime}}^{\left(j, j^{\prime}\right)} a_{j^{\prime} \nu^{\prime}},
$$

where $E_{0}$ is the resonance energy, the indices $j$ and $j^{\prime}$ label the particles, and $\left\langle j, j^{\prime}\right\rangle$ are the nearest neighbors. We are interested only in the states excited at the normal light incidence upon the zigzag plane $(x y)$; i.e., the mode polarization $\nu$ can be $x$ or $y$ for dipole resonances and $\nu=x z, y z$ for the quadrupole resonances. Hence, $V_{\nu \nu}^{\left(j, j^{\prime}\right)}=t_{\|} \cos ^{2} \psi+$ $t_{\perp} \sin ^{2} \psi$ (with $\nu=x$ or $x z$ ), $V_{\nu \nu}^{\left(j, j^{\prime}\right)}=t_{\|} \sin ^{2} \psi+t_{\perp} \cos ^{2} \psi$ (with $\nu=y$ or $y z$ ) and $V_{\nu \nu^{\prime}}^{\left(j, j^{\prime}\right)}=\left(t_{\|}-t_{\perp}\right) \sin \psi \cos \psi$ for $\nu \neq \nu^{\prime}$, where $\psi$ is the azimuth angle of the vector $\boldsymbol{R}=\boldsymbol{r}_{j}-\boldsymbol{r}_{j^{\prime}}$. Here, $t_{\|}$and $t_{\perp}$ are the coupling constants of 
(a)

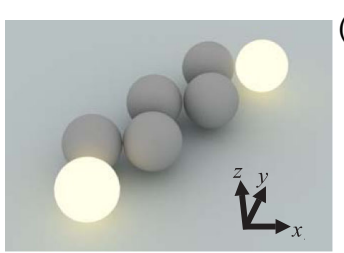

(b)

(c)

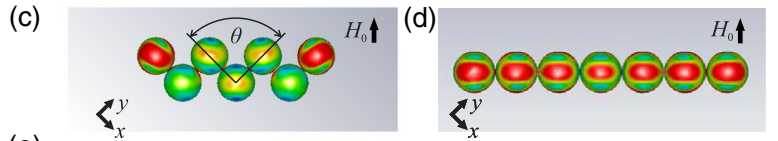

(e)

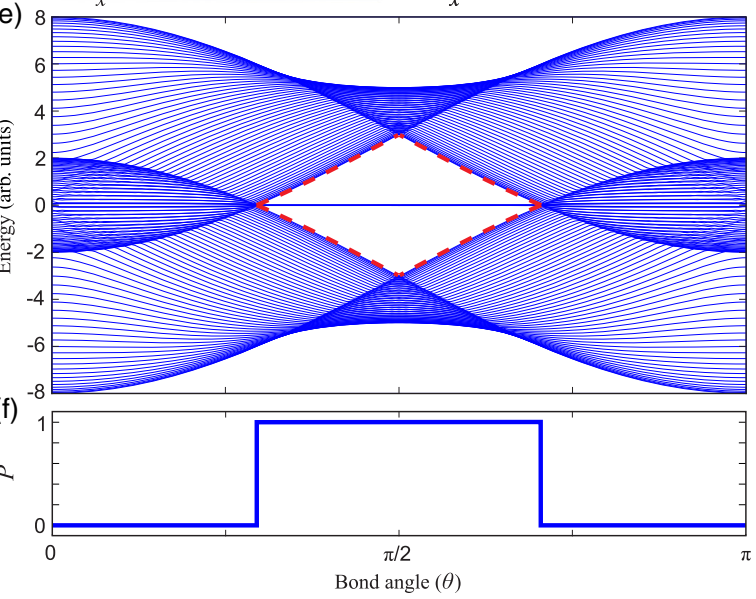

FIG. 1 (color online). Artist's view of (a) topologically nontrivial zigzag array and (b) topologically trivial linear chain of nanoparticles. (c),(d) Numerically calculated electric field distributions in both structures under the excitation by the plane wave with the direction of magnetic field $\boldsymbol{H}_{0}$ shown in the graphs. (e),(f) Energy spectrum and parity of the winding number $P \equiv W \bmod 2$ of the zigzag chain calculated as functions of the bond angle $\theta$. Red dashed line indicates the region when the edge states are formed. Calculated at $t_{\|}=4, t_{\perp}=-1, E_{0}=0$ for $N=60$ particles.

the modes copolarized and cross polarized with respect to the link $\boldsymbol{R}$; for quadrupole-quadrupole interaction $t_{\|} / t_{\perp}=-4$ and for dipole-dipole interaction $t_{\|} / t_{\perp}=-2$. The calculated spectrum of the Hamiltonian (1) for the finite chain with $N=60$ particles is shown in Fig. 1(e) as a function of the angle $\theta$ between two consecutive links in the zigzag. The value of $\theta$ equal to $\pi$ corresponds to a straight line when the two polarizations $\nu$ are decoupled, the system is not gapped and no edge states are present [Figs. 1(b), 1(d)]. The case $\theta \ll 1$ corresponds to a degenerate zigzag with very acute angles. For $|\theta-\pi / 2|<\arcsin |2 t / \Delta|$, a spectral gap appears with a pair of eigenstates, one localized at the left edge and one at the right edge [Figs. 1(a), 1(c)]. Their topological origin follows from the bulk $4 \times 4$ Hamiltonian

$$
H(k)=\left(\begin{array}{cc}
0 & Q \\
Q^{\dagger} & 0
\end{array}\right), \quad Q=h_{0}+\boldsymbol{h} \cdot \boldsymbol{\sigma}
$$

where $K$ is the Bloch wave vector, $h_{0}=\bar{t}\left(1+e^{-\mathrm{i} K}\right), h_{z}=$ $(\Delta / 2)\left(1+e^{-\mathrm{i} K} \cos 2 \phi\right), \quad h_{x}=(\Delta / 2) e^{-\mathrm{i} K} \sin 2 \phi, \quad h_{y}=0$,

$\bar{t}=\left(t_{\|}+t_{\perp}\right) / 2, \Delta=t_{\|}-t_{\perp}$. Equation (2) is written in the basis of the excitations on first and second particles in the unit cell; the Pauli matrices $\sigma$ act in the polarization space. The Hamiltonians Eq. (1) and Eq. (2) are chiral and belong to the chiral orthogonal class [17]. According to Refs. [18] and [19], this symmetry guarantees at least a pair of zeroenergy eigenstates when the winding number $W$ of $\operatorname{det} Q$, calculated when $K$ varies from $\pi$ to $-\pi$, is not equal to zero. The parity $P$ of the winding number is shown in Fig. 1(f). It is equal to one for $|\theta-\pi / 2|<\arcsin |2 t / \Delta|$ and plays a role of $Z_{2}$ topological index in our system [20]. Explicit derivations are given in the Supplemental Material [21]. In the nearestneighbor approximation, the edge states are robust against all perturbations respecting the chiral symmetry, including fluctuations of both coupling constants and link angles (Fig. S3 in [21]). We note, that for $t_{\|} t_{\perp}>0$ when $|\Delta|<2|t|$, the gap is open, and the edge states are present for all $\theta \neq 0, \pi$, see Fig. S4 in [21]. Such a situation could be realized for coupled micropillar cavities [22].

Topological edge states in our system strongly rely on polarization degeneracy of eigenmodes of individual particles, and thus, the problem is more general and much richer than the celebrated scalar Su-Schrieffer-Heeger (SSH) model for polyacetylene [23]. Although optical analogues of the SSH model were extensively studied in dimer superlattices [24-26], its topological properties have been fully understood only recently $[14,27,28]$. In our system, contrary to the SSH model, all the particles are the same and a pair of zero-energy states is present for both odd and even numbers of particles $N$. Localization degree increases with the number of particles [21]. For odd $N$ and $\theta=\pi / 2$ the edge states are copolarized, and for even $N$, they are cross polarized. This opens a possibility to selectively excite the left or right edge of the same structure by varying the incident wave polarization.

For our Letter, we choose dielectric spheres with large permittivity $(\varepsilon=15)$ as the building blocks (or "metaatoms") of our subwavelength electromagnetic structures [see Figs. 1(a), 1(b)]. From the exact Mie solution of light scattering by a spherical particle, the lowest frequency resonance in the spectra is the magnetic dipole resonance (at the frequency $\omega \approx c / R$ for $\varepsilon=15$, where $c$ is the speed of light), the second one is the electric dipole resonance $(\omega \approx 1.4 c / R)$, and the third one is the magnetic quadrupole resonance $(\omega \approx 1.5 c / R)$, see Ref. [29]. Based on the symmetry analysis above, the topologically nontrivial edge states are expected for both electric or magnetic Mie-type resonant modes, see also Fig. S5 in [21]. According to the multipole decomposition of a single dielectric sphere [see Fig. 2(a)], an obvious advantage of the magnetic quadrupole resonance is the high quality factor $(Q \sim 70)$ due to reduced radiative losses. The experimentally measured forward scattering amplitude of a single sphere is shown in Fig. 2(b). In agreement with the theoretical results in Fig. 2(a) based on the optical theorem, the experimental 

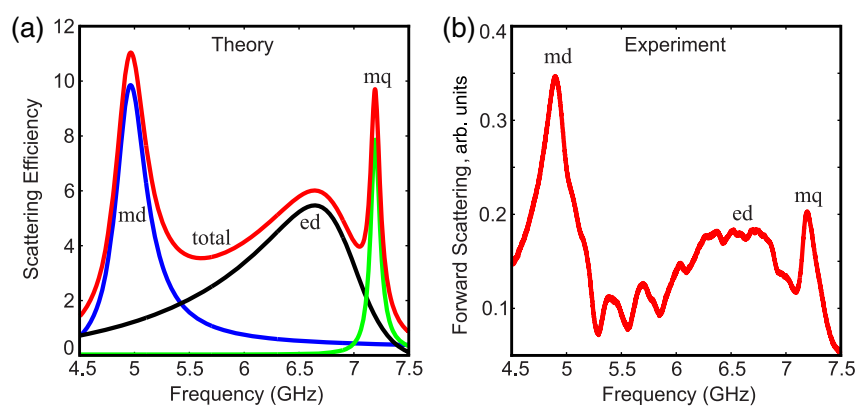

FIG. 2 (color online). (a) Scattering efficiency calculated by Mie theory, and (b) measured forward scattering of a dielectric sphere.

scattering spectrum demonstrates three maxima at the positions of magnetic dipole, electric dipole, and magnetic quadrupole resonances, marked on the graph. Figure 1 shows two types of dielectric arrays made from such spheres: a zigzag array with the bond angle $\theta=\pi / 2$, in Fig. 1(a), and a linear array, in Fig. 1(b). For both the arrays, the spheres are touching each other. Panels 1(c) and 1(d) show the numerically calculated electric field amplitude induced by a plane wave in both zigzag and linear structures at the frequency of the quadrupole resonance of the single sphere. In both cases, the incoming plane wave is polarized perpendicular to the array as indicated by an arrow for the magnetic field $\boldsymbol{H}_{0}$. The full-wave numerical simulations are performed using the CST MICROWAVE STUDIO 2014. The colors correspond to the absolute value of the electric field at the surface of the particle. The calculated field has strong maxima at the first and last particles in the zigzag array [see Fig. 1(c)]; however, the field distribution in the linear array is rather uniform and no edge states can be observed [see Fig. 1(d)].

Being inspired by this numerical demonstration of the edge excitation in the zigzag array, we have performed the proof-of-concept experiments in the microwave frequency range. To mimic the electromagnetic properties of silicon nanoparticles, we employed $\mathrm{MgO}-\mathrm{TiO}_{2}$ ceramic spheres that are characterized by a dielectric constant of 15 and small dielectric loss factor in the $4-10 \mathrm{GHz}$ frequency range [30]. The sphere radius is equal to $R=7.5 \mathrm{~mm}$ and the spheres are touching each other. Our main experimental results are summarized in Fig. 3. For chosen parameters, the magnetic quadrupole resonance frequency is equal to $f=7.2 \mathrm{GHz}$ [see Fig. 2(b)]. Next, we proceed to the analysis of the near-field maps measured in the close vicinity of the dielectric zigzag array at the magnetic quadrupole resonance [see Figs. 3(a)-3(d)]. In order to approximate the plane wave excitation, we utilize a rectangular horn antenna. It is connected to the transmitting port of a vector network analyzer (Agilent E8362C). We use an automatic mechanical near-field scanning device and an electric field probe connected to the receiving port of the analyzer. The probe is oriented normally with respect
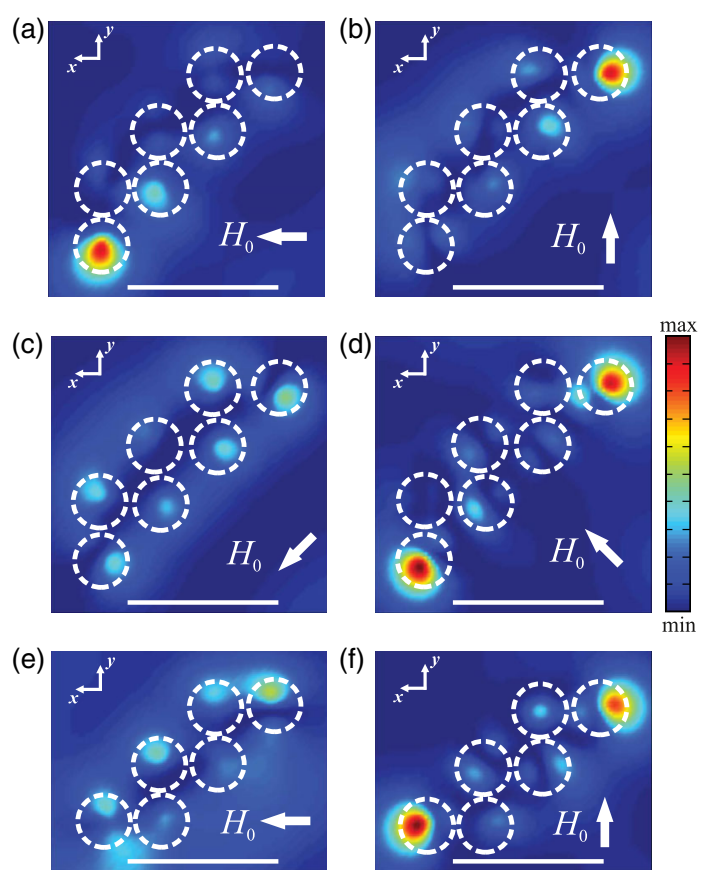

FIG. 3 (color online). Experimental results. (a)-(d) Measured electric field intensity at the frequency of the magnetic quadrupole resonance of a single sphere for different incident wave polarizations $\phi=0^{\circ}, 90^{\circ},-45^{\circ}$, and $45^{\circ}$, respectively, indicated in the panels by arrows. (e),(f) Electric field intensity measured at the $1 \mathrm{~mm}$ distance from the surface of particles for (e) $\phi=0^{\circ}$ and (f) $\phi=90^{\circ}$ polarizations. (a)-(d) Results for odd number of particles. (e)-(f) Results for even number of particles. White scale bar represents the incident wavelength.

to the interface of the structure, and we measure the $E_{z}$ component of the electric field. The near field was scanned at the $1 \mathrm{~mm}$ distance from the back interface of the zigzag array to avoid the contact between the probe and the sample. The polarization dependence of the structure response is examined by rotating the source antenna. Figures 3(a)-3(d) show the electric field maps for different angles $\phi$ between the polarization direction of the incident magnetic field $\boldsymbol{H}_{0}$ and the $x$ axis. The maps present a direct confirmation of the edge excitation in the structure at the magnetic quadrupole resonance. The relative intensity of the field above the edges can be switched by rotating the incident wave polarization. For 3 (a) $\phi=0^{\circ}$ or 3(b) $\phi=90^{\circ}$ polarizations, only the first or last sphere is excited, respectively, while for $3(\mathrm{~d}) \phi=45^{\circ}$ polarization, the induced electric fields are localized near both edges of the zigzag array. These results directly demonstrate the difference between our polarization sensitive system and the scalar SSH model. Based on the simplified analysis [15], the edge mode excitation scenario is different for an even number of particles, where both edges are excited identically with polarization-dependent intensities. Similar behavior is observed experimentally for six dielectric particles at the magnetic quadrupole resonance [see Figs. 3(e), 3(f)]. 
The experimental results of Fig. 3 are in full qualitative agreement with the predictions of the symmetry analysis of the Hamiltonian Eq. (1).

Further evidence of the presence of the topological edge states in our structure can be provided by an analytical model including the retarded coupling between the quadrupole modes and their Mie scattering on the spheres. The electric field outside the spheres is expanded over magnetic quadrupole modes

$$
\boldsymbol{E}(\boldsymbol{r})=\sum_{\boldsymbol{\rho}} \sum_{M= \pm 1} C_{\boldsymbol{\rho}, M} \boldsymbol{Y}_{2 M}^{(2)}(\boldsymbol{r}-\boldsymbol{\rho}) H_{2}^{(1)}(\omega|\boldsymbol{r}-\boldsymbol{\rho}| / c),
$$

where $\boldsymbol{\rho}$ is the sphere center position, $\boldsymbol{Y}_{2 M}^{(2)}$ is the transverse electric (TE) polarized spherical harmonics with the total and orbital momenta equal to 2 , and the azimuthal momentum $M$ [31], $H_{2}^{(1)}$ is the Hankel function of the first kind. The $x z$ and the $y z$ quadrupole modes can be expressed as the linear combinations $C_{\rho, x z}=\left(C_{\rho, 1}+C_{\rho,-1}\right) / \sqrt{2}, C_{\rho, y z}=$ $\left(C_{\rho, 1}-C_{\rho,-1}\right) /(\mathrm{i} \sqrt{2})$. The values of the expansion coefficients $C_{\rho, M}$ are obtained from the equation system

$$
C_{\boldsymbol{\rho}, M}=r_{2}^{(\mathrm{TE})}(\omega)\left(\xi_{M}^{(0)}+\sum_{M^{\prime}= \pm 1} \sum_{\rho^{\prime} \neq \boldsymbol{\rho}} G_{\rho M, \rho^{\prime} M^{\prime}}^{(\mathrm{TE}, \mathrm{TE})} C_{\rho^{\prime} M^{\prime}}\right) .
$$

Here, $r_{2}^{(\mathrm{TE})}(\omega)$ is the Mie reflection coefficient of the TE spherical wave from a sphere [32], $G_{\rho M, \rho^{\prime} M^{\prime}}^{(\mathrm{TE}, \mathrm{TE})}$ are the structure constants of the vector Korringa-Kohn-Rostocker method $[33,34]$ and $\xi_{M}^{(0)}=-4 \pi \boldsymbol{E}_{0}^{*} \cdot \boldsymbol{Y}_{2 M}^{(2)}\left(\boldsymbol{e}_{z}\right)$, where $\boldsymbol{E}_{0}$ is the electric field amplitude of the plane wave incident along the $z$ direction.

Figures 4(a)-4(f) show a comparison between our analytical results for the quadrupole-quadrupole model with the results of our full-wave numerical simulations. In Fig. 4(a), we plot the numerically calculated extinction cross section for the single sphere (red curve), for the zigzag chain when $\phi=45^{\circ}$ (blue dashed curve), and $\phi=0^{\circ}$ (black solid curve). The frequency of the magnetic quadrupole resonance of a single sphere is marked by the black dashed line. The spatial distributions of the electric field near the first and last dielectric particle are shown in Figs. 4(c)-4(f). At each frequency, we normalize the electric field above the first (\#1) and last (\#7) particle centers by the averaged field above the central particles (\#2-\#6). Both analytical and numerical calculations demonstrate strong resonant excitation of the edge spheres. The insets in Figs. 4(c)-4(f) show the electric field maps above the zigzag chain for different polarizations of the incident plane wave at the magnetic quadrupole resonance frequency. The polarization dependence of the effect and the field distributions perfectly agree with the experiment of Fig. 3. We also compare the electric field dependence on the particle number in numerical simulations, analytical model, and experiment [see Fig. 4(b)]. The agreement
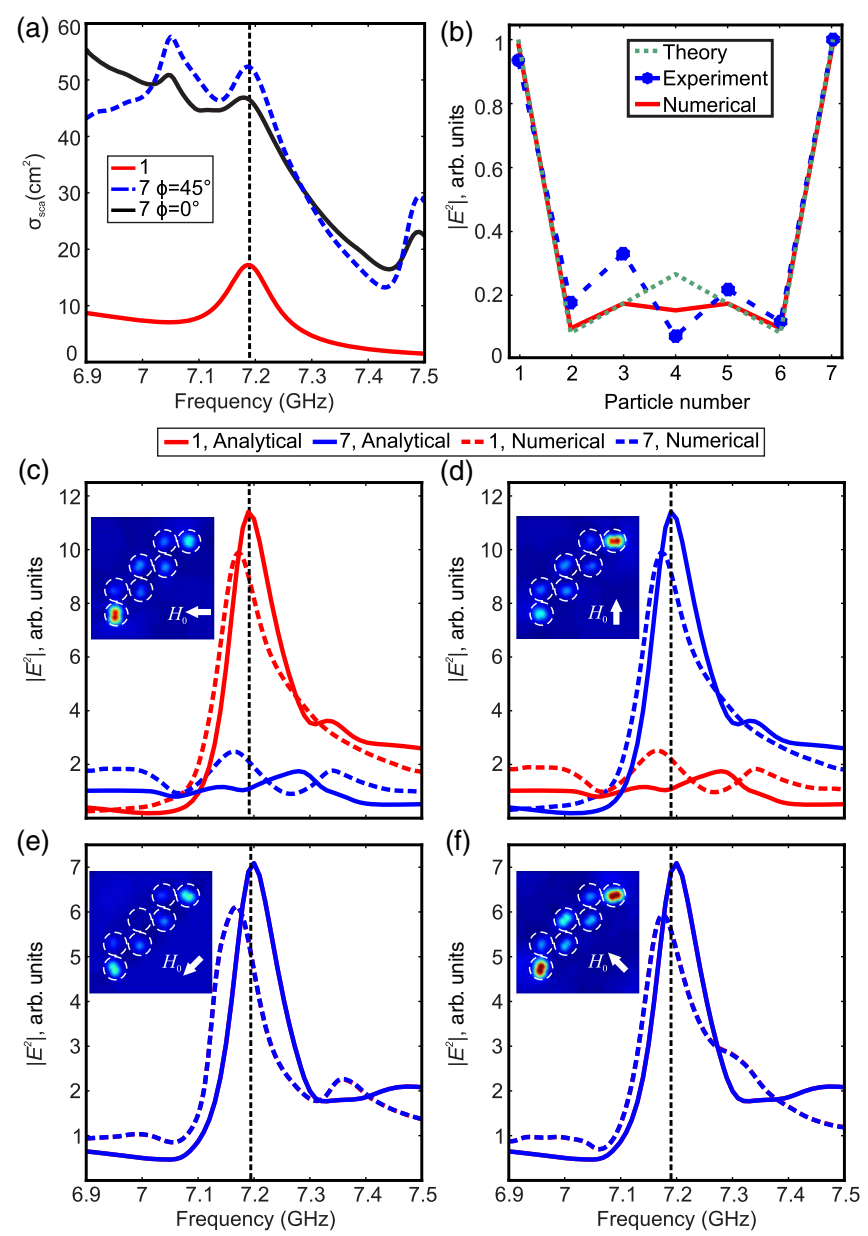

FIG. 4 (color online). (a) Extinction cross section spectrum for a single particle (red solid curve), and for the zigzag array at $\phi=45^{\circ}$ (blue dashed curve) and $\phi=0^{\circ}$ (black solid curve). (b) Dependence of the electric field near the particle center on the particle's number for $\phi=45^{\circ}$. (c)-(f) Near-field intensity at $1 \mathrm{~mm}$ above the first and last spheres for incident wave polarization $\phi=0^{\circ}, 90^{\circ},-45^{\circ}$, and $45^{\circ}$, respectively. Solid lines correspond to the analytical results and dashed lines to the results of numerical simulations. The insets correspond to the electric field intensity at the quadrupole magnetic resonance of the single particle (marked by a dashed black curve).

between the theoretical and experimental results is quite good. A small difference can be explained by the tolerance of dielectric particle manufacturing and small deviations of permittivities of different spheres. It should be mentioned that the experimental curve (blue line) in Fig. 4(b) corresponds to $\left|E_{z}\right|^{2}$, while the analytical (green line) and numerical (red line) curves correspond to $|E|^{2}$. In numerical simulations, we also observe the edge excitation when the incident wave is polarized with $\phi=-45^{\circ}$ [see Fig. 4(e)], while in the experiment this effect is not pronounced [see Fig. 3(c)]. This is due to the fact that, when $\phi=-45^{\circ}$, the amplitude of the induced fields is much weaker [see the inset in Fig. 4(e)] than in the case of 
excitation with $\phi=0^{\circ}, 90^{\circ}, 45^{\circ}$ [see the insets in Figs. 4(c), 4(d), and 4(f)].

In conclusion, we have theoretically proposed and experimentally demonstrated a novel class of photonic topological edge states realized in the zigzag arrays of subwavelength dielectric resonant structures. We have demonstrated, experimentally, selective excitation of topological edge states by adjusting the polarization of the incident wave, and we have clarified the difference between topologically trivial and nontrivial electromagnetic modes in such arrays. We believe that the study of topological polarization-entangled eigenmodes suggests a new way for engineering the properties of subwavelength structures and all-dielectric metamaterials for novel applications in nanophotonics.

We thank A. Khanikaev and D. Jacobs for useful discussions. This work was supported by the Australian Research Council, the Government of the Russian Federation (Grant No. 074-U01), the Dynasty Foundation (Russia), and the Russian Fund for the Basic Research. A.P.S. acknowledges a support of the SPIE scholarship. A. N.P. acknowledges a support of the President Grant of the Russian Federation (No. MK-6029.2014.2).

[1] M. Z. Hasan and C. L. Kane, Rev. Mod. Phys. 82, 3045 (2010).

[2] C. L. Kane and E. J. Mele, Phys. Rev. Lett. 95, 146802 (2005).

[3] B. A. Bernevig, T. L. Hughes, and S.-C. Zhang, Science 314, 1757 (2006).

[4] L. Lu, J. D. Joannopoulos, and M. Soljačić, Nat. Photonics 8, 821 (2014).

[5] K. Fang, Z. Yu, and S. Fan, Nat. Photonics 6, 782 (2012).

[6] A. B. Khanikaev, S. Hossein Mousavi, W.-K. Tse, M. Kargarian, A. H. MacDonald, and G. Shvets, Nat. Mater. 12, 233 (2013).

[7] M. C. Rechtsman, J. M. Zeuner, Y. Plotnik, Y. Lumer, D. Podolsky, F. Dreisow, S. Nolte, M. Segev, and A. Szameit, Nature (London) 496, 196 (2013).

[8] Z. Wang, Y. Chong, J. D. Joannopoulos, and M. Soljačić, Nature (London) 461, 772 (2009).

[9] W. Tan, Y. Sun, H. Chen, and S.-Q. Shen, Sci. Rep. 4, 3842 (2014).

[10] W.-J. Chen, S.-J. Jiang, X.-D. Chen, B. Zhu, L. Zhou, J.-W. Dong, and C. T. Chan, Nat. Commun. 5, 5782 (2014).

[11] M. Hafezi, S. Mittal, J. Fan, A. Migdall, and J. M. Taylor, Nat. Photonics 7, 1001 (2013).

[12] Y. E. Kraus, Y. Lahini, Z. Ringel, M. Verbin, and O. Zilberberg, Phys. Rev. Lett. 109, 106402 (2012).

[13] L.-J. Lang, X. Cai, and S. Chen, Phys. Rev. Lett. 108, 220401 (2012).
[14] M. Atala, M. Aidelsburger, J. T. Barreiro, D. Abanin, T. Kitagawa, E. Demler, and I. Bloch, Nat. Phys. 9, 795 (2013).

[15] A. Poddubny, A. Miroshnichenko, A. Slobozhanyuk, and Y. Kivshar, ACS Photonics 1, 101 (2014).

[16] M. R. Shcherbakov, D. N. Neshev, B. Hopkins, A. S. Shorokhov, I. Staude, E. V. Melik-Gaykazyan, M. Decker, A. A. Ezhov, A. E. Miroshnichenko, I. Brener, A. A. Fedyanin, and Y. S. Kivshar, Nano Lett. 14, 6488 (2014).

[17] S. Ryu, A. P. Schnyder, A. Furusaki, and A. W. W. Ludwig, New J. Phys. 12, 065010 (2010).

[18] S. Ryu and Y. Hatsugai, Phys. Rev. Lett. 89, 077002 (2002).

[19] M. Sato, Y. Tanaka, K. Yada, and T. Yokoyama, Phys. Rev. B 83, 224511 (2011).

[20] S. Ganeshan, K. Sun, and S. Das Sarma, Phys. Rev. Lett. 110, 180403 (2013).

[21] See Supplemental Material at http://link.aps.org/ supplemental/10.1103/PhysRevLett.114.123901 for details of (S1) analysis of the energy spectrum and topological properties; (S2) edge states at the dipole resonance; (S3) dependence on the number of particles; (S4) the role of material losses in the system, which includes Refs. [17], [19], [22].

[22] S. M. de Vasconcellos, A. Calvar, A. Dousse, J. Suffczyński, N. Dupuis, A. Lemaître, I. Sagnes, J. Bloch, P. Voisin, and P. Senellart, Appl. Phys. Lett. 99, 101103 (2011).

[23] S.-Q. Shen, Topological insulators. Dirac equation in condensed matters (Springer, Heidelberg, 2013).

[24] A. A. Sukhorukov and Y. S. Kivshar, Phys. Rev. Lett. 91, 113902 (2003).

[25] N. Malkova, I. Hromada, X. Wang, G. Bryant, and Z. Chen, Opt. Lett. 34, 1633 (2009).

[26] F. Dreisow, M. Heinrich, R. Keil, A. Tünnermann, S. Nolte, S. Longhi, and A. Szameit, Phys. Rev. Lett. 105, 143902 (2010).

[27] H. Schomerus, Opt. Lett. 38, 1912 (2013).

[28] X. Li, E. Zhao, and W. Vincent Liu, Nat. Commun. 4, 1523 (2013),

[29] A. I. Kuznetsov, A. E. Miroshnichenko, Y. H. Fu, J. Zhang, and B. Luk'yanchuk, Sci. Rep. 2, 492 (2012).

[30] R. S. Savelev, A. P. Slobozhanyuk, A. E. Miroshnichenko, Y.S. Kivshar, and P. A. Belov, Phys. Rev. B 89, 035435 (2014).

[31] D. A. Varshalovich, A. Moskalev, and V. Khersonksii, Quantum theory of angular momentum (World Scientific, Singapore, 1989).

[32] M. Born, E. Wolf, and A. Bhatia, Principles of optics: Electromagnetic theory of propagation, interference and diffraction of light (Cambridge University Press, Cambridge, England, 1999).

[33] X. Wang, X.-G. Zhang, Q. Yu, and B. N. Harmon, Phys. Rev. B 47, 4161 (1993).

[34] I. E. Psarobas, N. Stefanou, and A. Modinos, Phys. Rev. B 62, 278 (2000). 